\title{
Relación familia escuela \\ en contextos de pobreza. Posibilidades y limitaciones en los procesos educativos
}

\section{Family Relationship - School in contexts of poverty. Possibilities and limitations in educational processes}

Aceptación: 15 de marzo de 2010.

Alejandra Santana López ${ }^{1}$ Aprobación: 15 de junio de 2010.

\section{RESUMEN}

El artículo trata sobre la reflexión respecto la vinculación familia-escuela en contextos de pobreza, el análisis es orientado por la hipótesis de que esta relación puede ser positiva o negativa según la incidencia que tenga en los niños, en sus aprendizajes y en el entorno educativo. El desarrollo del análisis se da mediante la revisión de la literatura, abordando tres caminos para la reflexión: la perspectiva de la efectividad escolar, la perspectiva de la sociedad del conocimiento y el cambio educativo y la perspectiva sociocultural. Desde esta reflexión preliminar se esbozan algunas sugerencias de mejora para los establecimientos, señalando estrategias específicas según los distintos actores que el contexto educativo convoca.

Palabras clave: Relación parental, pobreza, escuela, participación paterna.

\begin{abstract}
This paper focuses on a reflection on family ties-School in contexts of poverty, the analysis is guided by the assumption that this relationship can be positive or negative depending on the impact that has on children in their learning and the learning environment. The development of the analysis is given by reviewing the literature, addressing three paths for reflection: the perspective of school effectiveness, the perspective of the knowledge society and educational change and the prospect socio - cultural. From this preliminary discussion outlines some suggestions for improvements to facilities, pointing out specific strategies for different actors who call the educational context.
\end{abstract}

Key words: Parental relationship, poverty, school, parental involvement.

Trabajadora Social, Magíster en Psicología, Doctor ( e en Ciencias de la Educación. Docente Escuela de Trabajo Social Universidad Andrés Bello, Santiago. Correo: asantana@uc.cl 


\section{Presentación}

El presente artículo plantea, preliminarmente, una reflexión respecto a la relación Familia - Escuela ${ }^{2}$ en los procesos de aprendizajes de los niños en contextos de vulnerabilidad social, caracterizado por situaciones de pobreza, con especial consideración del contexto chileno. La hipótesis a la base señalaría que esta relación puede estimarse como positiva o negativa según la incidencia que pueda tener en el niño, sus aprendizajes y el entorno educativo al que pertenecen. Este análisis pretende revisar el estado del arte, desde las investigaciones en el área. Para esto se realizó la revisión de fuentes bibliográficas, procurando desarrollar una discusión desde los autores, tendiendo a marcar diferencias entre las diversas perspectivas de abordaje al tema. El texto se organiza en cuatro apartados. El primero contextualiza la situación de la Educación en Chile, apuntando a la calidad y equidad en establecimientos a los accede la población más pobre del país. En el segundo apartado se desarrollan tres perspectivas de análisis, que posibilitan la comprensión de la relación Familia-Escuela, estas son: la perspectiva de la efectividad escolar, la perspectiva de la sociedad del conocimiento y el cambio educativo; y, finalmente, la perspectiva sociocultural. El tercer apartado, plantea sugerencias de acción a la política educativa desde la investigación en esta área. Diferenciando estrategias de acción según ámbito o actor al cual apunta de manera más directa, pues se entiende que todos están sistémicamente conectados.

El cuarto apartado, ofrece una reflexión final a modo de conclusión, donde se sintetizan los aspectos centrales del análisis.
1. La calidad de la educación en Chile ¿qué pasa con las escuelas pobres?

Al entender la educación como un derecho, debiesen los Estados garantizarla a todos sus ciudadanos y a todos con los mismos estándares de calidad, eso sería lo justo y equitativo. Sin embargo, en Chile esto no es así, "las escuelas que atienden a los dos quintiles más pobres alcanzan rendimientos muy bajos. En promedio no logran que sus alumnos lean y calculen bien” (Eyzaguirre, 2004).

La calidad de la educación ha sido uno de los temas que ha convocado múltiples debates en Chile, país donde la cobertura está garantizada, sin embargo donde la calidad no ha tenido una presencia equitativa en las escuelas, más bien se ha presentado conforme a las brechas socioeconómicas en que se emplazan. Así se pueden ver resultados positivos en establecimientos particulares -pagados y particulares - subvencionados, sin embargo, la educación municipal - gratuita reviste graves debilidades en distintos ámbitos de la calidad.

A más de 15 años de la puesta en marcha de la Reforma Educativa chilena, "las brechas de equidad permanecen o se acentúan y la calidad no mejora significativamente a pesar de las inversiones y esfuerzos realizados" (Muñoz, 2004, 114), lo que se hace visibles desde las mediciones nacionales, especialmente $\mathrm{SIMCE}^{3}$ y $\mathrm{PSU}^{4}$, por nombrar las de mayor cobertura territorial en el país.

Las inequidades en acceso y calidad en educación corresponden a un problema social, al cual intentan responder políticas públicas; pero por sobre todo es un imperativo ético. Para la investigadora Ruth Lupton (2005), alcanzar justicia social en educación, requiere como mínimo que todos los estudiantes tengan acceso a escuelas de la misma calidad, aun cuando sus resultados sean desiguales; habitualmente las escuelas ubicadas en ba-

Se entenderá una noción amplia y diversa de familia, que dé cabida a las nuevas configuraciones familiares: familias monoparentales, familias recompuestas, familias extensas, entre otras.

3 Sistema Nacional de Evaluación de Aprendizaje del Ministerio de Educación de Chile.

4 Prueba de Selección Universitaria - Chile. 
rrios más pobres comúnmente son juzgadas por otorgar una menor calidad que aquellas que están sectores más aventajados.

En relación a la inequidad, esta "se presenta en distintas dimensiones y ellas se potencian, es decir, cuando más de una de ellas está presente, los problemas educativos de las comunidades se presentan de manera más fuerte 0 , bien, persisten problemas ya resueltos en comunidades con mejores condiciones socioeconómicas. Todos los estudios realizados, tanto en nuestra región como en otras regiones del mundo, muestran que la principal causa de fracaso escolar es la pobreza. Hay otras causas, pero la pobreza es la principal" (Machado, 2004, 17).

Estas reflexiones ubican varios temas sobre el tapete: derechos, políticas públicas, sociales, administración y gestión de la política educativa, la realidad de las escuelas, perfiles directivos, características del profesorado, perfiles de niños y jóvenes, familias y comunidades que conforman este sistema. Llama particularmente la atención y urge conocer las realidades en que las condiciones de pobreza y vulnerabilidad social limitan las posibilidades de un incremento en la calidad de la educación y en la forma en que esta se instala en los constructos culturales de comunidades y familias.

Desde este escenario parece relevante el papel que pueden jugar las familias, particularmente los padres $^{5}$, en incrementar la calidad de la educación de sus hijos. ¿Cómo pretender mejorar otras áreas de la educación, si los niños en situación de pobreza pertenecen a espacios de fuerte influencia social y simbólica como lo es la familia? ¿Cómo potenciar acciones sinérgicas que favorezcan la calidad de la educación desde una mirada que se centre en los recursos de la familia, más allá de su situación de pobreza? ¿Qué posibilidades de dar sustento a un cambio educativo que abogue por la calidad educativa incluyendo a los actores clave? ¿De qué manera la tensión con la familia puede ser una fuente de crecimiento en el marco del cambio educativo?

\section{Perspectivas de análisis a la relación familia-escuela}

La relación Familia - Escuela está situada sociohistóricamente, ha ido cambiando conforme ha cambiado la sociedad en su conjunto. "Las primeras escuelas mantenían una estrecha unión con la comunidad. A principios del s. XX, comenzaron a distanciarse. La labor pedagógica se fue especializando y haciendo cada vez más compleja y los maestros enseñaban materias y utilizaban métodos alejados de la experiencia de los padres y madres, que poco tenían que decir acerca de lo que ocurría en el interior de las aulas" (Maestre, 2009, 6). Este alejamiento se mantuvo, incluso, argumentando que Familia y Escuela buscaban objetivos divergentes. Hoy se plantea que tienen influencias superpuestas, responsabilidades compartidas, teniendo que redefinir la relación bajo condiciones de colaboración (op. cit.).

En general, se destaca una influencia positiva asociada a la colaboración entre Familia y Escuela, mejorando la autoestima de los niños, el rendimiento escolar, las relaciones entre padres e hijos, las actitudes de los padres hacia la escuela y como consecuencia una escuela y una educación más eficaz y de mayor calidad (Scout-Jones, 1995; Epstein \& Sander, 2000; Vélez, 2009).

Sin embargo, las visiones no son unívocas, desde la investigación educativa distintas perspectivas de análisis son más o menos propensas a incorporar a las familias en los procesos educativos en los contextos de pobreza o vulnerabilidad.

Para aproximarse a una revisión del estado del arte en torno a la relación Familia-Escuela, se abordarán tres perspectivas de análisis, las cuales conciben la relación de la familia con la escuela desde prismas diversos, aportando matices que van

Para efectos de este documento la actoría social de la familia, se manifestará en la acción de los padres. Entendiendo por padres a las personas que cumplen la función de tales, pudiendo ser biológicos, adoptivos, sustitutos, entre otros. 
desde visiones que plantean -eventualmente- la exclusión de los padres en la tarea formativa, dado su escaso aporte a los procesos de aprendizajes, versus miradas que hacen apuestas centradas en la comprensión de las dimensiones culturales de las familias, apuntando a prácticas familiares que puedan potenciar los aprendizajes de los niños, entendiendo que esta relación se encuentra situada social, cultural e históricamente.

\subsection{La perspectiva de la efectividad escolar: el "efecto familia"}

Para comprender a qué apunta la perspectiva de la efectividad escolar, se debe conocer qué se entiende por una escuela efectiva, esta "promueve de forma duradera el desarrollo integral de todos y cada uno de sus alumnos más allá de lo que sería previsible teniendo en cuenta su rendimiento inicial y su situación social, cultural y económica" (Murillo, 2003 en Bellei et col, 2004). Agregando que, "para ser eficaz la escuela debe favorecer el desarrollo de todos y cada uno de sus alumnos y estos deben progresar más de lo que es esperable conforme a las características socioeconómicas y culturales de su familia. A veces se agrega como criterio que la escuela eficaz se preocupe por el desarrollo integral del alumno, esto es, además de buenos resultados de aprendizaje, se preocupe de su formación en valores, bienestar y satisfacción, desarrolle toda la personalidad de los alumnos" (op. cit., 2004, 1).

Para la perspectiva de la efectividad escolar, de la relación de factores que explican los resultados escolares por el logro de aprendizaje, aquellos que tienen mayor peso son: el entorno familiar de los alumnos, la calidad de la comunidad donde residen y la efectividad de la escuela. Para los países desarrollados, los estudios atribuyen un peso de un $80 \%$ a la familia y la comunidad. En cambio, en las sociedades en vías de desarrollo, debido a su grado más alto de desigualdad, la escuela está llamada a compensar las diferencias de origen sociofamiliar, pudiendo incidir más enérgicamente en los resultados escolares (Brunner \& Elacqua, 2004). Pero, ¿igualmente las sociedades en vías de desarrollo deben considerar a los padres en los procesos de aprendizajes de los niños?, esta posibilidad de incidir de manera compensatoria ¿no tendría un impacto mayor si reconoce e integra a la familia y a la comunidad?

Así, desde el reconocimiento de los factores que explican los logros de aprendizajes, la influencia que tiene la familia se le ha denominado "el efecto familia", "suele pensarse que este factor estaría determinado exclusivamente por el nivel de ingreso del hogar. En verdad, como muestran los estudios, este factor es mucho más complejo que eso. Tiene que ver con el "mundo de vida" en que nace y se desarrolla el niño. Pero aún más decisivo parece ser, en el caso de niños provenientes de hogares de escasos recursos, la organización de la familia, su clima afectivo, la socialización lingüística o la adquisición temprana de actitudes y motivaciones" (op. cit., 2004).

El "efecto familia" apela a los recursos básicos en la conformación de las personas, a la experiencia de socialización primaria y a las posibilidades culturales que tiene la familia, en lenguaje de Bourdieu (1986), respondería a los capitales previos que posee la familia, los cuales son condicionantes de la posibilidad de adquisición futura o activación de otros capitales, tales como los sociales y culturales, a los que se pueda optar durante el desarrollo de la persona, en su niñez, juventud y adultez.

En relación a este legado familiar, Eyzaguirre (2004) plantea que "en el ambiente educativo circula la noción de que la falta de apoyo de los padres, la ausencia de capital cultural de las familias, la baja capacidad intelectual, las carencias de lenguaje, los problemas de aprendizaje, que se perciben como prevalentes en los sectores pobres, impedirían alcanzar altos rendimientos. Si bien la evidencia indica que estos factores son condicionantes, no muestra que sean determinantes" (p.252). Cabe destacar, que aunque la perspectiva de la efectividad escolar atribuye una ponderación alta al "afecto familia", en la práctica no lo considera, proponiendo incluso mantenerlo al margen de los procesos impulsados por los establecimientos.

Ahora bien, como ya se señalaba el "efecto familia" no se reduce al ingreso fa- 
miliar o a la escolaridad de los padres, este ha sido desagregado, recopilando la visión de diversos investigadores (Buchmann, 2003; Levin \& Belfield, 2002; Marzano, 2000; Sheerens, 2000 en Brunner \& Elacqua, 2004), en un listado de variables de entorno familiar y escolar que inciden en los logros de aprendizaje, estas son:

- Ocupación, ingreso y nivel educacional de los padres.

- Infraestructura física del hogar y grado de hacinamiento.

- Recursos del hogar (libros, diccionarios, escritorio, computadora).

- Organización familiar y clima afectivo del hogar.

- Alimentación y salud durante los primeros años de vida del niño.

- Prácticas de socialización temprana.

- Desarrollo lingüístico y tipo de conversaciones en el hogar.

- Rutinas diarias, desarrollo de actitudes y motivación.

- Acceso a y calidad de, la enseñanza preescolar.

- Elección de escuela.

- Armonía entre códigos culturales de la familia y la escuela.

- Estrategia de aprendizaje y conocimiento previo adquiridos.

- Involucramiento familiar en tareas escolares.

- Uso del tiempo en el hogar y durante las vacaciones.

Para potenciar la efectividad escolar en contextos de pobreza, Bárbara Eyzaguirre (2004), identifica líneas estratégicas para lograr escuelas efectivas y que obtengan buen rendimiento, estas apuntarían a: "la importancia de tener expectativas y exigencias altas, de presionar hacia el logro, de un liderazgo fuerte hacia lo académico, de definir metas académicas claras y concretas que sean el foco de la escuela, de concentrarse en la enseñanza y el aprendizaje, de focalizarse en las destrezas básicas, de crear un ambiente de aprendizaje y trabajo entre los profesores, de la presencia regular de pruebas externa rigurosas, el monitoreo del progreso, la noción de que el tiempo dedicado al estudio es clave en el aprendizaje y de que el esfuerzo crea la habilidad, la importancia del uso efectivo del tiempo, la importancia de un ambiente seguro y ordenado que facilite el aprendizaje, el compromiso y participación de los padres"(p.251). Paradójicamente, al momento de sugerir acciones específicas con los padres deja entrever el riesgo de tomar mucho tiempo y energía en el trabajo con ellos, siendo incluso más adecuado mantenerlos al margen e intentar que los niños encuentren satisfacción a necesidades de pertenencia, afectivas y de socialización en el mismo establecimiento.

\subsection{La perspectiva de la sociedad del conocimiento en un contexto de cambio educativo}

Una segunda visión, la perspectiva de la sociedad del conocimiento como una forma de comprender los procesos educativos, ha sido desarrollada por Hargreaves (2003), quien a través de múltiples paradojas del sistema explica lo que se espera de las nuevas formas de organización escolar, articulando con enseñanza efectiva y aspectos pedagógicos en el marco de procesos de transformación educativa o reformas. Esta mirada tiene múltiples puntos de encuentro con la perspectiva de efectividad escolar, sin embargo se optó por presentarla de manera diferenciada, ya que propone un rol específico para la familia en situación de pobreza, concibiéndolos como consumidores de un servicio.

Así, en relación a características particulares que las familias en situación de pobreza pudiesen presentar y que afecten la relación colaborativa entre Familia y Escuela, se identifican: la ausencia de los padres como actores fundamentales en la formación de sus hijos, dificultades familiares diversas que influyen en la condición de salud biopsicosocial de los niños, recursos socioculturales escasos y/o ausencia de conciencia de la relevancia de la educación para sus hijos (Lupton, 2005., Stevenson \& Stingler, 1999, Eyzaguirre, 2004; Fullan \& Stiegelbauer, 2003; Fullan, 2002).

Aparentemente, la participación de Familia en contextos vulnerables se visualiza, más bien, como un obstaculizador. Para ilustrar los diversos roles, posibilidades o limitaciones que los padres pueden jugar en la escuela, Hargreaves (2003) señala que en la 
sociedad del conocimiento, donde escuelas y docentes corren en función de alcanzar resultados en pruebas estandarizadas, la relación con los padres se reduce a "transacciones mercantiles en las que las escuelas tratan a los padres como consumidores, bien a reacciones defensivas que consideran a los padres como elementos de interferencia a través de las quejas" (p.98).

Las dificultades directas o indirectas con las familias de los niños, son reportadas también en el estudio de Stevenson \& Stingler (1999), donde los profesores norteamericanos planteaban las dificultades en el proceso de enseñan a los niños, donde las dificultades familiares operaban como obstaculizadores, "familias que viven con un solo progenitor, madres que trabajan y no tienen tiempo de atender a sus hijos, niños que después de la escuela tienen que ir a algún tipo de guardería a esperar que sus madres vayan a buscarlos; niños que tienen llaves de su hogar y entran a una casa vacía a la espera de que llegue el progenitor. Los alumnos tienen tantos problemas personales y familiares que a duras penas pueden salir adelante (...) Esta clase de problemas rebasa lo que pueden hacer el maestro o la escuela: solo pueden resolverse a nivel de la familia y de la sociedad" (p.332).

Lupton (2005) pone el acento en padres que no tienen instalado el valor de la educación, lo que podría explicarse por sus propias vivencias escolares. Señala que en contextos vulnerables, resulta especialmente "problemático" que, la evaluación de las escuelas acerca de la contribución de los padres al aprendizaje de los alumnos en la escuela y en la casa, ya que asegurar que los padres contribuyan a los aprendizajes de los alumnos es cuantitativa y cualitativamente diferente en escuelas donde la mayoría de los padres están motivados y preparados lo suficiente como para aportar, que en escuelas donde muchos padres no han tenido una experiencia favorable en las escuelas, no están familiarizados con el sistema escolar y no están convencidos del valor de la educación que sus hijos están recibiendo.

Para Fullan (2002), respecto a la relación cambio educativo e incorporación de los padres, señala que cuanto más cerca esté el padre de la educación del niño, tanto mayor será el impacto en la evolución y logros educativos del niño" (Fullan \& Stiegelbauer, 2003, 195). Aun cuando la evidencia es un tanto confusa, "una de las razones porque es tan confuso el papel de los padres es la de que con frecuencia no se define específicamente, lo que se entiende por participación, ni se vincula esta con resultados particulares (...) es indudable que los antecedentes familiares y la situación en el hogar implican una diferencia. Los estudiantes de las familias más privilegiadas (en término de condiciones en el hogar que apoyan el aprendizaje) se desempeñan mejor en la escuela" (Fullan \& Stiegelbauer, 2003, 196).

En relación a las posibilidades de participación de los padres en el contexto escolar, Maestre (2009) identifica dos áreas: en el aula y en el establecimiento en general. En el aula incluye: entrevistas, apoyo en casa a las tareas escolares, acompañantes de salidas, participación en talleres de diversa índole, participación en asambleas de clase o etapa y fiestas y celebraciones. De la participación en el establecimiento, se considera: en el consejo escolar, en las asociaciones de padres y apoderados, en escuelas para padres y en fiestas, celebraciones o exposiciones de la escuela.

Por otro lado, la visión de Fullan \& Stiegelbauer (2003), respecto a la participación de los padres en el proceso de cambio educativo, identifica como principales formas de participación: la participación paterna en la escuela (voluntariados, asistentes); participación paterna en las actividades de aprendizaje en casa (ayuda a los niños en el hogar, clases particulares); relación hogar - comunidad - escuela (comunicación), y gobierno (consejos consultores).

\subsection{Perspectiva sociocultural: capital sim- bólico y cultural de las familias}

La perspectiva sociocultural se centra en el reconocimiento de la crianza, los vínculos de socialización de las familias y la forma en que esto media la relación Familia - Escuela, dimensión que impregna a la "persona" del niño, ya que opera en su conformación como tal. Por lo tanto, no es viable afectar 
los aprendizajes de los niños sin considerar esta dimensión constitutiva de su ser.

Esta perspectiva asume que los padres poseen creencias y expectativas respecto a sus hijos y a los procesos educativos en que están involucrados, tienen estilos de crianzas que afectan la forma en que participan y se vinculan a la Escuela. Lo que quiebra la idea que todos los padres se relacionan de la misma manera con la Escuela.

Annette Lareau (2000) ha estudiado la relación entre clase social y educación, desde esta concepción la clase social se asocia a espacios de diferenciación, que permiten explicar desigualdades, generadores de identidad, que se vinculan a grupos ampliados, es decir, se conforman de acuerdo a las relaciones que se dan ese espacio.

Esta perspectiva, parte del reconocimiento que la pertenencia a una clase social tiene una poderosa influencia en la vida de las familias y en la vida de los niños fuera del hogar, sin embargo, se reconocen múltiples vacíos en la investigación en esta área, por ejemplo, se desconoce bajo qué circunstancias la clase social le da a los niños ventajas poderosas, y bajo cuáles la ventaja es trivial o no existe (Lareau, 2000).

La diferencia en el grado de participación de los padres, desde una perspectiva cultural, se podría comprender desde el trabajo de Bourdieu y su concepto de capital cultural, así las escuelas elaborarían de forma desigual los recursos sociales y culturales en la sociedad. Bajo esta lógica, se enfatiza la importancia de la estructura del colegio y de la familia y las disposiciones de los individuos en la comprensión de los distintos niveles de participación parental en la Escuela (op. cit., 2000).

De esta forma se pueden reconocer al menos dos visiones, desde las familias: comunidades de clases trabajadoras y las de clase media alta. En el caso de los padres de clase trabajadora, ellos creen que los profesores son los responsables de la educación, respetan y confían en su experiencia, eventualmente buscan consejos de parte de ellos, buscan poca información acerca del currículo o de los procesos educacionales y sus críti- cas a la escuela se centran en aspectos no académicos. En definitiva, estos padres más bien mantienen separada la relación familia escuela, en vez de conectada (Lareau, 2003).

Por otra parte, los padres de clase media - alta, establecen relaciones de control y de interconexión entre la vida familiar y escolar, creen que la educación es una responsabilidad compartida entre profesores y padres, son críticos con la escuela y se informan frente a ella.

Otras diferencias que destaca la autora apuntan a las habilidades y recursos para mejorar el rendimiento de los hijos, donde los padres de clase alta tienen mayor educación, estatus e ingreso, lo que aumentaría sus competencias para ayudar a sus hijos. La vinculación entre padres, parientes y amigos los hace estar más informados respectos a los procesos educativos.

Sin embargo, cabe destacar que ambos grupos de padres comparten el deseo de éxito de sus niños en las escuelas (Lareau, 2000).

En esta misma línea, Elina Dabas (2005) destaca la fuerza que tiene la familia en tanto fuente de múltiples interacción internas y hacia su medio “(...) en todas, sus diferentes miembros participan en diversos contextos, lo cual modifica implícita y explícitamente las posibilidades de aprendizaje de sus integrantes, al enriquecer cada una de las interacciones con las experiencias realizadas en los diversos ámbitos de acción. La información que los niños aportan provenientes del ámbito escolar o de las casas de sus compañeros; los comentarios que los padres reciben en su trabajo acerca de modalidades de vida y de crianza influencian y modifican cada una de las relaciones cotidianas" (Dabas, 2005, 31 ), lo que se relaciona con las disposiciones familiares de capital cultural y social, las que cambian según clase social.

Así, por ejemplo, hay "familias que poseen valoraciones diferentes de la escuela y que por lo tanto varían tanto sus expectativas hacia el éxito de los hijos como la ayuda que brindan con respecto a la demanda de la escuela. También observamos diferencias con respecto a la participación y a las relaciones 
que establecen los docentes" (Dabas, 2005, 31). Por su parte, existen "familias que elaboran diferentes estrategias ante el fracaso escolar de los hijos. Otras tienen altas expectativas con respecto al éxito escolar pero que sus comportamientos reales no coinciden con ellas" (op. cit., 31). Valoraciones y expectativas son parte de los aspectos que son contenidos en la matriz sociocultural de las familias.
Para sintetizar estas tres perspectivas de análisis asociadas a la relación Familia-Escuela, se presentará la siguiente Tabla (Tabla No1) que buscar ofrecer una mirada comparada -en líneas gruesas - de las tres miradas, especificando las representaciones subyacentes de: los niños, los padres, los profesores, la escuela en su conjunto y la relación Familia - Escuela.

Tabla No 1

Síntesis Perspectivas de Análisis Relación Familia-Escuela en contextos de pobreza

\begin{tabular}{|c|c|c|c|}
\hline $\begin{array}{l}\text { Representaciones } 1 / \\
\text { Perspectivas de } \\
\text { Análisis }\end{array}$ & $\begin{array}{l}\text { Perspectiva de la } \\
\text { Efectividad Escolar } \\
\text { (Eyzaguirre, 2004; } \\
\text { Buchman, 2003; } \\
\text { Levin \& Belfield, } \\
\text { 2002; Brunner \& } \\
\text { Elacqua, 2004) }\end{array}$ & $\begin{array}{l}\text { Perspectiva de la sociedad } \\
\text { del conocimiento en } \\
\text { contexto de cambio } \\
\text { educativo } \\
\text { (Hargreaves, 2003; } \\
\text { Lupto, 2005; Stevenson \& } \\
\text { Stingler, 1999; Fullan \& } \\
\text { Stiegelbauer, 2003) }\end{array}$ & $\begin{array}{l}\text { Perspectiva sociocultural } \\
\text { (Lareau, 2000: Lareau, 2003; } \\
\text { Dabas, 2005) }\end{array}$ \\
\hline $\begin{array}{l}\text { Representación de } \\
\text { los niños }\end{array}$ & $\begin{array}{l}\text { Sin referentes } \\
\text { de socialización, } \\
\text { afectos y } \\
\text { pertenencia. }\end{array}$ & $\begin{array}{l}\text { Con problemas } \\
\text { psicosociales personales y } \\
\text { familiares. } \\
\text { Poseen escasos recursos } \\
\text { culturales. }\end{array}$ & $\begin{array}{l}\text { Persona en proceso de } \\
\text { socialización. } \\
\text { Pertenece a una clase social. }\end{array}$ \\
\hline $\begin{array}{l}\text { Representación de } \\
\text { los padres }\end{array}$ & $\begin{array}{l}\text { Afectan el } \\
\text { rendimiento } \\
\text { escolar. } \\
\text { Se requiere su } \\
\text { compromiso y } \\
\text { participación. } \\
\text { Eventualmente hay } \\
\text { que excluirlos. } \\
\text { Conforman parte } \\
\text { del efecto familia. }\end{array}$ & $\begin{array}{l}\text { Consumidores de un } \\
\text { servicio. } \\
\text { Eventualmente interfieren } \\
\text { con sus quejas. } \\
\text { Son fuente de dificultad } \\
\text { para la tarea educativa. } \\
\text { No están conscientes } \\
\text { de la relevancia de la } \\
\text { educación. } \\
\text { Son obstaculizadores para } \\
\text { el cambio. }\end{array}$ & $\begin{array}{l}\text { Son heterogéneos. } \\
\text { Agentes claves de } \\
\text { socialización. } \\
\text { Son parte del capital } \\
\text { simbólico de la familia. } \\
\text { Poseen creencias y } \\
\text { expectativas respecto a } \\
\text { educación. } \\
\text { Pertenecen a una clase } \\
\text { social en que construyen una } \\
\text { identidad. } \\
\text { Poseen determinadas } \\
\text { disposiciones frente a los } \\
\text { procesos educativos. }\end{array}$ \\
\hline
\end{tabular}

Se habla de Representación aludiendo a las nociones, ideas, construcciones que desprenden directamente de los autores referidos o que se pueden inferir de ellos, la tabla busca organizar las visiones a partir de la revisión bibliográfica correspondiente. 
Tabla No 1

continuación

\begin{tabular}{|c|c|c|c|}
\hline $\begin{array}{l}\text { Representación de } \\
\text { los profesores }\end{array}$ & $\begin{array}{l}\text { Deben entrenarse } \\
\text { y entrenar para } \\
\text { responder a los } \\
\text { estándares. } \\
\text { Deben generar } \\
\text { ambientes de } \\
\text { trabajo. } \\
\text { Deben ejercer } \\
\text { liderazgo } \\
\text { académico. }\end{array}$ & $\begin{array}{l}\text { Deben centrarse en } \\
\text { trabajar para obtener } \\
\text { resultados. }\end{array}$ & $\begin{array}{l}\text { Son los expertos en el área de } \\
\text { la educación. } \\
\text { Prestan consejo y apoyo. } \\
\text { Poseen creencias y } \\
\text { expectativa de los niños, sus } \\
\text { familias y las posibilidades de } \\
\text { desarrollo de estos. }\end{array}$ \\
\hline $\begin{array}{l}\text { Representación de } \\
\text { la escuela }\end{array}$ & $\begin{array}{l}\text { Debe ser efectiva } \\
\text { Altas exigencias } \\
\text { para ellas } \\
\text { Se debe fortalecer } \\
\text { la propuesta } \\
\text { educativa. } \\
\text { Eventualmente } \\
\text { compensa lo que } \\
\text { los padres no } \\
\text { aportan. }\end{array}$ & $\begin{array}{l}\text { Debe centrarse en trabajar } \\
\text { para obtener resultados. }\end{array}$ & $\begin{array}{l}\text { Instancia que elaboraría de } \\
\text { forma desigual los recursos } \\
\text { sociales y culturales de la } \\
\text { sociedad. }\end{array}$ \\
\hline $\begin{array}{l}\text { Representación de } \\
\text { la relación familia- } \\
\text { escuela }\end{array}$ & $\begin{array}{l}\text { Efecto, que de ser } \\
\text { positivo se integra } \\
\text { a la formación. } \\
\text { Efecto, que de ser } \\
\text { negativo se excluye } \\
\text { y es compensado } \\
\text { por la escuela. }\end{array}$ & $\begin{array}{l}\text { Espacio para transacciones } \\
\text { mercantiles del servicio } \\
\text { educativo. Donde la } \\
\text { familia es un consumidor. }\end{array}$ & $\begin{array}{l}\text { Relaciones institucionales, } \\
\text { discontinuas y desconectadas. }\end{array}$ \\
\hline
\end{tabular}

3. Estrategias de apoyo que se desprenden de las perspectivas de investigación en la relación familia-escuela en contextos de pobreza

Reconociendo que los aportes de la investigación educativa en materia de vinculación familia-escuela, se agrega a los insumos que sustenta la política educativa, a continuación se señalan algunas sugerencias para la acción.

Estas sugerencias de acción se presentan como ámbitos que deben recibir apoyo desde el planteamiento de la política educativa para potenciar los procesos educativos en contextos de pobreza.
Son múltiples las sugerencias dadas a la acción de la política educativa para contextos de vulnerabilidad y pobreza, algunas de ellas van en la línea de: políticas de mejoramiento, diversificadas, localmente codiseñadas, más recursos, coordinación de servicios públicos, pedagógicamente diversa.

También se deberían estimar recursos para apoyo extra para niños en desventaja o contrataciones de las áreas psicosociales, que puedan ser un soporte interno de las dificultades de los niños, pero también un articulador de la red local de servicios. En este sentido abogar, más bien, por estrategias de mejoramiento contextualizado que permita recoger la complejidad del escenario sociocultural 
donde se vive la escuela. Lo que es coherente con las indicaciones de Lupton (2005), quien después de realizar un estudio con escuelas en situación de pobreza recomienda estrategias de mejoramiento basadas en las apreciaciones del contexto, considerando factores locales, sociales, demográficos y económicos, el mejoramiento escolar y la historia institucional. Esto, además, sería consistente con el hecho de que las familias en situación de pobreza acceden a las escuelas que están en sus entornos inmediatos, en el caso chileno, establecimientos municipales; lo que exige coordinación a nivel de gobierno local y eficiente canalización de los recursos para ello.

La participación de los padres y su real incorporación a los aprendizajes de sus hijos se debiese enmarcar en iniciativas de mejoramiento globales al sistema de educativo, como parte de las estrategias de cambio educativo contextualizado, es decir, tomando en consideración las variables locales de diversas índole, entre ellas las características de la comunidad y las familias de origen de los niños. De lo contrario se corre el riesgo de actuar sobre un ámbito de manera lineal, unicausal, homogénea y sin impactar en los aprendizajes, en la calidad de ellos y en transformaciones culturales que se puedan sostener y proyectar en el tiempo.

Claramente, una estrategia de mejora a los aprendizajes que incluya a los padres debe contemplar la red social inmediata de la escuela, las dimensiones culturales y todo recurso ecológico que puede estar al servicio del cambio. “(...) Las familias, las escuelas y las diversas organizaciones de la comunidad pueden mejorar su interrelación para contribuir a un mejor proceso educativo, ya que el cambio tecnológico y cultural no pasa necesariamente por la escuela en sí misma sino que acontece dentro y fuera de ella, sin poder delimitar claramente dónde comienza uno y otro"(Dabas, 2005, 35).

\section{Apoyando a los niños}

Desde la perspectiva de la efectividad escolar se plantea que los niños son centrales en las estrategias de apoyo. "Si en pobreza son más frecuentes los hogares poco estructurados, con menor ascendiente y preocupación de los padres por la educación formal de sus hijos, la escuela tiene que crear lazos personales con los niños. El alumno debe sentir que en las escuelas hay personas a quienes les importa su presencia, su bienestar, su futuro y su comportamiento. Aparte de lo que esto significa para sentirse importante y querido, interesa porque los niños se mueven para responder por un futuro abstracto" (Eyzaguirre, 2004, 272), llegando con esto a compensar el rol de los padres.

Lo anterior implica, especialmente, en condiciones extremas de abandono o negligencia parental, constituir una fuente referencial para el niño, un recurso no solo para sus aprendizajes sino un recurso que lo permita sostenerse humanamente. En este sentido un rol crucial tienen los profesores, quienes tienen una interacción más sistemática y recurrente con los niños.

Otras posibilidades de apoyo, más centradas en dimensiones culturales aportarían a fortalecer ámbitos de socialización de los niños, sin anular o negar su propia construcción de padres y familia a los que pertenece, en estrecha vinculación con un trabajo en paralelo con los padres.

\section{Apoyando a los padres}

Si se optara por enfocar parte de las acciones de apoyo a la efectividad de los aprendizajes y a la calidad de estos en los padres como agentes claves en respaldar los procesos de aprendizajes de los niños desde sus hogares, se evidencia que la literatura no es enfática en señalar la ventaja de esta línea de acción. Aun cuando parte importante de los autores ve la vinculación padres - escuela como una díada positiva, pocos hacen la salvedad, de las particularidades que el apoyo a los padre debería tener con las familias que viven en situaciones de vulnerabilidad social.

Desde el enfoque cultural es clave trabajar con los padres, no solo en estrategias que fortalezcan sus habilidades parentales, sino abordando el sentido que tiene para ellos, la educación de sus hijos, cómo se ha construido este sentido desde sus experiencias educacionales y cómo lo proyectan en sus 
hijos en la actualidad. Lo anterior, a fin de gatillar cambios en sus propias disposiciones y acciones en su rol, respecto a la educación de los niños.

\section{Apoyando a los profesores}

Desde la efectividad escolar, en casos extremos se ofrece "sortear" a las familias y padres "poco colaboradores" de los procesos de aprendizaje de los niños. En este sentido se sugiere que "los profesores deben atreverse a exigir y deben resistir los reclamos de alumnos y padres. Tiene que haber conciencia de que el paternalismo y la sobreprotección son dañinos" (Eyzaguirre, 2004, 268).

En esta misma línea, la falta de colaboración efectiva de parte de los padres no puede obstaculizar la tarea educativa y la escuela se debe esforzar en ello, por ejemplo, si los niños no hacen las tareas porque sus padres no los apoyan, lo peor que se puede hacer es suspenderlas, hay que buscar soluciones creativas, tanto en el fortalecimiento de tutores o con el fomento de autorresponsabilidad, enseñar a los padres o instituir espacios en el colegio para que los niños se queden y puedan realizarlas (Op. cit.).

Desde la perspectiva cultural resulta relevante en la tarea de apoyar a los profesores, explicitar sus propias creencias y expectativas en relación a las familias y a los niños en contextos de pobreza; la literatura ha sido clara en señalar que estas creencias afectan directamente los procesos de enseñanzaaprendizajes desarrollados por los profesores.

Por otra parte, se debe destacar que no es competencia de los profesores el abordaje de problemáticas psicosociales que puedan presentarse en las familias en situación de pobreza, por lo que se hace necesario el diálogo interdisciplinario, el contar con agentes expertos (al interior o al exterior de la comunidad educativa) con quienes dialogar y el compartir criterios comunes a la base de las acciones emprendidas.

\section{Apoyando a la Escuela}

A nivel de escuela, la propuesta de Lupton (2005) apunta a proveer recursos para distintos diseños organizacionales, que permitan mayor calidad para ser entregada en forma consistente en circunstancia de alta pobreza, recursos que debieran darse en reconocimiento a un trabajo diferente y no como un financiamiento aparte o extra al que hay que postular.

Desde la mirada de la efectividad, la escuela se debe concentrar en lo académico, si hay problemas de índole familiar que están interfiriendo, la recomendación sería evaluar la posibilidad de contratar a un profesional especializado que canalice los problemas de los niños y sus familias a las instancias adecuadas, permitiendo que profesores y directivos se centren en lo académico (Eyzaguirre, 2004, 263).

Por su parte, desde la visión sociocultural, destacaría la noción de clase social como espacio social de diferenciación en las relaciones sociales, en este marco la escuela conforma una comunidad educativa abierta a su medio. Desde ahí deben emprenderse los cambios, especialmente, favoreciendo la conformación de redes sociales y la activación de estos, como estrategia clave para enfrentar contextos de pobreza.

\section{Conclusiones}

La educación en contextos de pobreza debe resguardar estándares de calidad que se traduzca en aprendizajes efectivos, significativos y con proyecciones respecto a los niños y jóvenes que son parte del sistema, como un imperativo ético que responde a criterios de justicia social. Sin embargo, es inviable diseñar una política educativa que apunte a mejoras que solo se centren en los niños y sus rendimientos, aislándolo de su entorno significativo. En este sentido, este documento pretendió revisar la literatura y los aportes en torno a la relación familia-escuela.

De esta forma se llegaron a diferenciar tres perspectivas de análisis desde la investigación educativa, que conciben de manera diversa la ingerencia de la familia en los procesos de aprendizaje de los niños, y, por ende, se muestran más o menos proclives a incluirla como agente social en los procesos de aprendizajes en los contextos de pobreza, 
lo que está sujeto a la naturaleza de sus concepciones de educación, familia y escuela.

A partir de estas vertientes de investigación se esbozaron recomendaciones a la política educativa, precisando líneas de acción para las escuelas y los actores: niños, padres y profesores.

Fruto de la revisión del estado del arte de la relación familia-escuela en contexto de pobreza, es posible establecer -exploratoriamente- algunas reflexiones.

La relevancia de la familia en los procesos de aprendizaje es una idea transversal e indiscutible en las visiones analizadas, ya sea considerando su efecto directo en los aprendizajes, su participación en las transformaciones educativas o como capital cultural y social de los niños.

La única perspectiva que llega a estimar la injerencia de manera más sólida teórica y metodológicamente hablando, es la perspectiva de la efectividad, quienes han estimado, relativamente, el "efecto familia", en alrededor de un $80 \%$ respecto a los efectos en los aprendizajes.

Lo que no es conclusivo es de qué manera trabajar con las familias, considerar o no una participación activa de estas, qué estrategias son pertinentes a los contextos de pobreza, en que las familias presentan vivencias multiproblemáticas, entre otros ámbitos. De esta forma se reconocen estrategias que van desde el polo de excluir a las familias de los procesos de aprendizajes hasta el polo de reeducar desde parámetros normativos a la familia, de tal forma que sea funcional al sistema educativo, pero muchas veces anulándola como actor legítimo de estos procesos.

A juicio de la autora, la perspectiva sociocultural ofrece amplias posibilidades de desarrollar nuevas líneas de investigación, de hecho Lareau $(2000,2003)$ plantea la necesidad de realizar investigaciones de carácter cualitativo que permitan iluminar la "caja negra" de la relación familia-escuela. Desde donde se puedan explorar dimensiones valóricas, creencias, perfiles de familias, estilos de vidas, identificación de recursos o capitales culturales, trayectorias particulares de experiencias de padres, condiciones de vida y dinámicas familiares, estilos parentales, entre otros; que en relación con los contextos escolares y determinados marcos de política educativa a nivel nacional e internacional aporten a la reducción de las inequidades sociales, por tanto, a la superación de la pobreza.

\section{Bibliografía}

BELLEI, C., MUÑOZ, G., PÉREZ, L., \& RACZYNSKI, D. (2003). Escuelas efectivas en sectores de pobreza. ¿Quién dijo que no se puede? Santiago: UNICEF - Asesorías para el Desarrollo.

BOURDIEU, P. (1989). Forms of Capital. En: Halsey, A., Lauder, H., Brown, P. \& Wells, A. (Eds.) (1997) Education: culture, economy, society. Oxford - New York University.

BRUNNER, J. \& ELACQUA, G. (2004). Factores que inciden en una educación efectiva. Evidencia internacional. Manuscrito. Santiago: Universidad Adolfo Ibáñez.

DABAS, E. (2005). Redes sociales, familias y escuela. Buenos Aires: Paidós.

EPSTEIN, J. \& SANDER, M. (2000). Handbook of the sociologic of Education. Ed. Springer: United State.

EYZAGUIRRE, B. (2004). Claves para la educación en pobreza. En: Estudios Públicos, 93 (verano).

FULLAN, M. (2002). Los nuevos significados del cambio en la Educación. Barcelona: Octaedro.

FULLAN, M. \& STIEGIELBAUER, S. (2003). El cambio educativo. Guía de planeación para maestros. México: Trillas.

GARCÍA - HUIDOBRO, J. (2004). Escuelas de calidad en condiciones de pobreza. Santiago: Universidad Alberto Hurtado \& BID.

HARGREAVES, A. (2003). Enseñar en la sociedad del conocimiento. Barcelona: Octaedro.

LAPTON, R. (2005). Social justice and school improvement: improving the quality of 
schooling in the poorest neighborhoods. British Educational Research Journal, 31 (5): 589-604.

LAREAU, A. (2000). Home advantage. Social class and parental intervention. Maryland: Rowman \& Littlefield Publishers, INC.

LAREAU, A. (2003). Unequal childhoods. Class, race, and family life. California: University of California Press.

MAESTRE, A. (2009). Familia y Escuela. Los pilares de la educación. En: Renovación y Experiencias Educativas, 14 (enero).
SCOTT - JONES, D. (1995). Parent - Chile Interactions and School Achievement. En: B. Ryan, G. Adams, T. Gullota, R. Weissberg \& R. Hampton (Eds). The Family - School Connetion. London: SAGE Publications.

STEVENSON, H. \& STIGLER, J. (1999). ¿Por qué los escolares de Asia Oriental tiene alto rendimiento académico? En: Estudios Públicos, 76 (primavera).

VÉLEZ, R. (2009). La relación familia - escuela como alianza. Aproximaciones a su comprensión e indagación [versión electrónica]. Revista educación, innovación, tecnología, 3 (6): 1-15. 